\title{
Evolutionary study of the Be star 28 Tau
}

\author{
Nazhatulshima Ahmad ${ }^{1}$, Mohd. Zambri Zainuddin ${ }^{1}$, \\ Mohd. Sahar Yahya ${ }^{2}$, Peter P. Eggleton ${ }^{3}$ and Hakim L. Malasan ${ }^{4}$ \\ ${ }^{1}$ Physics Dept., Faculty of Science, University of Malaya, 50603 Kuala Lumpur, Malaysia \\ ${ }^{2}$ Math. Div., Center of Fundamental Sciences, University of Malaya \\ ${ }^{3}$ Lawrence Livermore National Laboratory, US Livermore CA 945510808 United State \\ ${ }^{4}$ Astronomy Division, FMIPA, ITB, Jl. Ganesa 10, Bandung 40132 Indonesia
}

\begin{abstract}
We present an evolutionary study of 28 Tau, a Be star, in connection with its rapid rotation. The photometric data during the absence of its envelope in 1921 have been used to determine the effective temperature and luminosity of the star at the main sequence of the HR diagram. From an evolutionary model, we found that the mass and radius of the star are about $3.2 \mathrm{M}_{\odot}$ and $3.2 \mathrm{R}_{\odot}$ respectively. The equatorial rotation velocity of the star, $\nu_{e}$ found to be close to its critical velocity, $\nu_{c r}$ where $\nu_{e} / \nu_{c r} \simeq 0.87$.
\end{abstract}

Keywords. stars: fundamental parameters, stars: evolution, stars: rotation

\section{Introduction}

28 Tau (Pleione, BU Tau, HD 23862, HR 1180), a B8Vpe star (Hoffleit, 1995), is a well-known typical example of a Be star observed since the $18^{\text {th }}$ century. The variation of Be phases of 28 Tau has been reported since the early $19^{\text {th }}$ century until 2007 (Goraya et al., 1990, Katahira et al., 1996, Tanaka et al., 2007). The hydrogen emission lines were absent for about 32 years and have returned in late 1938 as a noticeable emission line with a fine central reversal (McLaughlin, 1938). The absence of hydrogen emission lines which indicate the destruction of the circumstellar matter - influences the measurement of the surface brightness of the star. In this study, photometric data during the absence of the envelope have been used to determine the effective temperature and luminosity of the star on the HR diagram. The evolutionary tracks were created using Eggleton code (Eggleton \& Kiseleva-Eggleton 2002) with consideration of the star's rotation.

\section{The evolutionary model}

The evolutionary track has been created at specific mass and rotation, taking into account the dynamo activity, mass loss by dynamo-driven stellar wind and magnetic braking, and metallicity $\mathrm{Z}=0.02 .28$ Tau has been found to be a spectroscopic binary by Katahira et al. (1996) with the secondary star expected to be a low-mass helium star or white dwarf. He concluded that the circumstellar gas around the primary star is supplied by the primary itself, based on the mass-loss rate of its companion. Hence, in this study we only evolve 28 Tau as a single star. With an initial mass, $\mathrm{M}=3.2 \mathrm{M}_{\odot}$ and rotational period, $\mathrm{P}_{\text {rot }}=0.334$ days, we found the best approximated values with $\log T_{\text {eff }}=4.0359$ and $\log L=2.1077$, based on photometric data during the absence of its envelope using the bolometric flux method (Gray, 1992) and the correlation of $\mathrm{T}_{\text {eff }}$ and BC (Flower, 1996). Table 2 shows the age in Myr, rotational velocity in $\mathrm{km} / \mathrm{s}$, critical velocity in $\mathrm{km} / \mathrm{s}$ and the ratio of rotational and critical velocities of each model in Fig 1 . Model 3 is an estimated position of 28 Tau on the HR diagram observed in 1921 where the ratio of 
Table 1. The photometric data taken from Binnendijk (1949) only using photovisual and Pv method. The bold data were chosen for this study in obtaining the $T_{\text {eff }}$ and $L$ of 28 Tau.

\begin{tabular}{|l|l|l|l|l|l|l|l|l|}
\hline Year & $\mathbf{m}_{v}$ & Met & Year & $\mathbf{m}_{v}$ & Met & Year & $\mathbf{m}_{v}$ & Met \\
\hline 1911 & $4.87 \pm 0.004$ & $\mathrm{Pv}$ & $1914-1915$ & $4.95 \pm 0.04$ & $\mathrm{v}$ & 1928 & $5.00 \pm 0.06$ & $\mathrm{Pv}$ \\
1913 & $4.99 \pm 0.05$ & $\mathrm{Pv}$ & $1916-1917$ & $4.94 \pm 0.04$ & $\mathrm{Pv}$ & 1931 & $4.96 \pm 0.06$ & $\mathrm{Pv}$ \\
$1914-1915$ & $4.92 \pm 0.03$ & $\mathrm{Pv}$ & $\mathbf{1 9 2 1}$ & $\mathbf{4 . 8 5} \pm \mathbf{0 . 0 3}$ & $\mathbf{P v}$ & 1936 & $5.04 \pm 0.04$ & $\mathrm{Pg}$ \\
\hline
\end{tabular}

Notes: Method : Pv-photovisual, v-visual and Pg-photographic

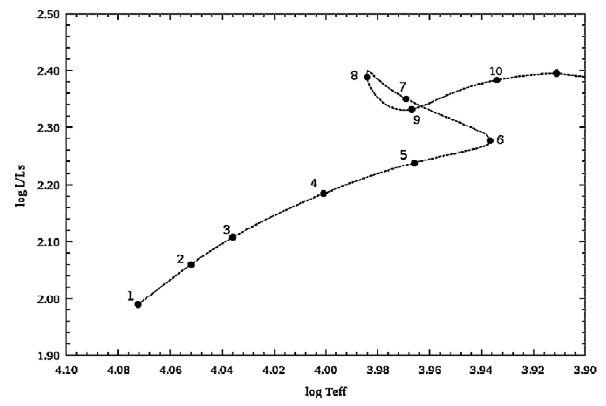

Figure 1. The evolutionary model of 28 Tau with initial $\mathrm{M}=3.2 \mathrm{M}_{\odot}$ and $\mathrm{P}_{\text {rot }}=0.334$ days.

Table 2. The values of age, $\mathrm{P}_{r o t}(\mathrm{~d})$, rotational velocity, $\nu$, and critical velocity, $\nu_{c r}$, at each model of the evolutionary track in Fig 1.

\begin{tabular}{c|c|c|c|c|c|c|c|c|c|}
\hline Model & Age & $\nu_{e}$ & $\nu_{c r}$ & $\nu_{e} / \nu_{c r}$ & Model & Age & $\nu_{e}$ & $\nu_{c r}$ & $\nu_{e} / \nu_{c r} \mid$ \\
\hline 1 & 0.01 & 347.02 & 458.55 & 0.76 & 6 & 316.24 & 202.23 & 283.04 & 0.71 \\
2 & 99.0 & 343.93 & 420.44 & 0.82 & 7 & 321.75 & 231.48 & 292.38 & 0.79 \\
$\mathbf{3}$ & $\mathbf{1 5 4 . 7 3}$ & $\mathbf{3 4 1 . 2 6}$ & $\mathbf{3 9 4 . 0 1}$ & $\mathbf{0 . 8 7}$ & 8 & 322.01 & 256.01 & 296.21 & 0.86 \\
4 & 232.20 & 320.28 & 347.74 & 0.92 & 9 & 322.09 & 230.66 & 294.06 & 0.78 \\
5 & 283.85 & 263.46 & 310.30 & 0.85 & 10 & 322.26 & 218.32 & 264.69 & 0.82 \\
\hline
\end{tabular}

rotational to critical velocities is 0.87 . The critical velocity is given by $\nu_{c r}=\sqrt{G M_{\star} / R_{e}}$ $=\sqrt{2 G M_{\star} / 3 R_{p}}$ where $R_{e}$ and $R_{p}$ are the radius at equator and pole respectively.

\section{Result and discussion}

The ratio of rotational velocity of 28 Tau to its critical velocity during the main sequence band has been calculated in the range of 0.71-0.92. The upper limit is close to the ratio by Townsend et al. (2004) i.e 0.95. From the models we suggest that the current mass of 28 Tau is $3.19 \mathrm{M}_{\odot}$, radius of $3.2 \mathrm{R}_{\odot}$ and rotational period is 0.475 days.

\section{References}

Binnendijk, L. 1949, AJ, 54, 117B

Eggleton, P. P. \& Kiseleva-Eggleton, L. 2002, ApJ, 575, 461

Flower, P. J. 1996, ApJ, 469, 355

Gray, D. F. 1992, The observation and analysis of stellar photospheres, Cambridge Astrophys. Ser., Vol. 20, p. 337

Goraya, P. S., Sharma, S. D., Malhi, J. S., \& Tur, N. S. 1990, Ap\&SS, 174, 1-11

Hoffleit, D. \& Warren, Jr., W. H. 1995, Bright Star Catalogue, 5th Revised Ed

Katahira, J.-I., Hirata, R., Ito, M., Katoh, M. et al. 1996, PASJ, 48, 317

McLaughlin, D. B. 1938, ApJ, 88, 622

Tanaka, K., Sadakane, K., Narusawa, S.-Y., Naito, H. et al. 2007, PASJ, 59, L35

Townsend, R. H. D., Owocki, S. P., \& Howarth, I. D. 2004, MNRAS, 350, 189-195 